\title{
Experimental System for Determining the Magnetic Losses of Super Paramagnetic Materials; Planning, Realization and Testing
}

\author{
Miloš Beković and Anton Hamler \\ University of Maribor, Faculty of Electrical Engineering and Computer Science, \\ Slovenia
}

\section{Introduction}

In recent decades we have witnessed the development of nanotechnology throughout all fields of science including the field of magnetic materials. When it comes to nanotechnology is usual for at least one of the dimensions to be in $\mathrm{nm}$ scale and therefore the nano-materials or nano-equipment exhibit certain unique characteristics. The area of magnetic materials also followed the developments within this field, with the focus on magnetic nanoparticles, which are typically super-paramagnetic. Within the context of nanotechnology, it is necessary to observe particles in comparison with solid magnetic materials because the unique characteristics enable some completely new applications.

Within this section, the magnetic fluid is exposed, as a representative of nano-materials, because of its unique features of super-paramagnetic properties and liquid state. The merger of these two properties is enabled because of the magnetic nanoparticles, as discussed in details in the second chapter. At this point several technical and biomedical applications can be listed and the benefits arising from the unique properties of magnetic fluids. Example applications in engineering mechanics regarding rotary sealing have been described by the authors (Jibin et al., 1992), whilst the biomedical use of these materials enabled new methods in medical diagnostics and therapeutic methods (Kumar, 2009). In this context the method of medical hyperthermia has been introduced, which is becoming a promising alternative treatment for cancerous tissues in contrast to the use of conventional methods (Barba et al., 2010; Pavel \& Stancu, 2009; Pollert, et al., 2007). The aforementioned method exploits the phenomenon of warming ferrofluids within alternating magnetic field with the aim of the thermally destroying cancer cells. This paper presents a physical mechanism responsible for the heating of magnetic fluid within a magnetic field, as well as the realization of an experimental system heating evaluation.

If modern medicine actually succeeds its usage of magnetic fluid for hyperthermia treatment then one of the important parameters is certainly its heating power. In reality, this is about the magnetic losses of magnetic materials in alternating magnetic field but in the context of a heating element for application within medical hyperthermia regarding heating power. Therefore, the precise characterization of magnetic losses is crucial and due to the specific properties of this material differing from conventional approaches, for determining 
any losses. This requires a measurement system that can determine these losses, this being the essence of this article. The following shows the design of such a system and its realization, as well as the actual testing on a magnetic fluid sample.

\section{Magnetic losses}

Magnetic fluids consist of stable colloidal dispersions of magnetic nanoparticles within a carrier liquid, where the Brownian motion keeps the particles permanently suspended, whilst adsorbed long-chain species on the particle's surfaces prevent their agglomeration (Rosensweig, 1997). Their physical properties are governed by the usage of carrier liquid whilst the magnetic properties are dependent on the types, sizes and concentrations of magnetic nanoparticles.

If such a fluid is exposed to an alternating magnetic field, its magnetic particles tend to align in the direction of the magnetic field, also known as magnetic relaxation. Each particle alignment is accompanied by energy consumption from the magnetic field, which is outwardly manifested as magnetic losses. Since these fluids usually contain a great number of particles and the rotations are frequent, as in the case of high-frequency fields, then the operation of the dissipation mechanisms elevates the temperature of the material. On the other hand, the amount of heat released within one magnetization cycle is equal to the area of the hysteresis loop (Carrey, et al., 2011, Beković, 2010).

These two properties of the fluid within the alternating magnetic field serve as the basis for determining of its heating power. The calorimetric method, where the determination of losses is based on the elevated temperature according to the equation below, is where the specific absorption rate (SAR) is determined as

$$
\mathrm{SAR}=\frac{\rho \mathrm{C}_{\mathrm{s}}}{m_{\mathrm{Fe}}}\left(\frac{\Delta T}{\Delta t}\right)_{t=0},
$$

where $\rho$ is the density of the fluid, $C_{\mathrm{s}}$ is the sample specific heat-capacity, $m_{\mathrm{Fe}}$ is the total content of the maghemite $\left(\mathrm{g}_{\mathrm{Fe}} / \mathrm{cm}^{3}\right.$ sample), and $\Delta T / \Delta t$ is the temperature change, respectively the initial slope of the measured temperature rise curve. In order to determine any loss by this method, in addition to the constant parameters of the liquid, measured temperature response is required, which varies depending on the amplitude and frequency of applied magnetic field.

The second method provides specific heating power (SHP) or magnetic losses $P_{\mathrm{h}}$ by determining the surface of the $B H$ hysteresis loop, as given by the equation

$$
\mathrm{SHP}=\frac{f}{\rho} \int_{0}^{T} H(t) \frac{\mathrm{d} B(t)}{\mathrm{d} t} \mathrm{~d} t,
$$

where the $H(t)$ is the magnetic field strength, $B(t)$ is the measured magnetic induction and $f$ the frequency of of magnetic field. Both these constants can be used for calculating the specific heating power $p$ in $\mathrm{W} / \mathrm{mm}^{3}$ according to (3), where $\varphi$ is mass density of the magnetic particles.

$$
p=\operatorname{SAR} \rho \varphi=\operatorname{SHP} \rho
$$


Both presented approaches provide an equivalent loss, as demonstrated in (3), whilst equations (1) and (2) serve as the base for designing a measurement system.

\section{Experimental system}

The basic idea for designing this experimental system was determining the magnetic losses of the magnetic fluid exposed to the magnetic field. The primary need for the realization of such a system is the generation of alternating magnetic fields with the possibility of changing the amplitude and frequencies of the magnetic fields, and the container for the measured sample. The specific needs of a measuring system for determining losses using the chosen method where determined according to equations (1) and (2). In the case of calorimetric methods, the temperature of the sample must be measured in order to determine the derivative $(\Delta T / \Delta t)$, whilst in case of the magnetic measurements method the time-dependent magnetic field strength and magnetic induction must be evaluate in order to determine the integral in (2). Other constants appearing in equations (1) and (2) are within the domain of the fluid's manufacturer of and are usually known.

\subsection{The planning}

The construction of the system, the design of which can be seen in the figure below (Fig. 1), was compiled from the results carefully planning its electrical and thermal properties. The figure only shows the basic elements of the system, but thereafter it is represented by the result of analyse which has resulted in a decision in the design and selected materials.

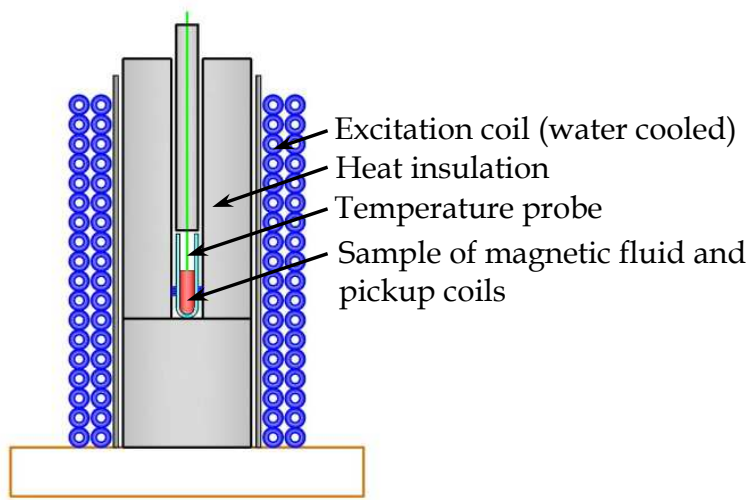

Fig. 1. The scheme of the key elements of the measurement system for the characterization of magnetic fluid heating power.

The measurement system was incorporated within a measurement scheme as seen in Fig. 2. which shows it's crucial elements. It is evident that a function generator is used for power amplifier control, by generating a sinus waveform of various amplitudes and frequencies. The power amplifier (Amplifier research) supplies an $L C$ circuit, where $L$ is the inductance of the excitation coil, and $C$ serves to create resonance conditions at a selected frequency. The temperature measurements are performed using two sensors, fibre optic and resistance. The measurements are performed using an oscilloscope, whilst control and data acquisition 
is done via the LabView software package; communication being performed via standard GPIB and RS 232 busses.

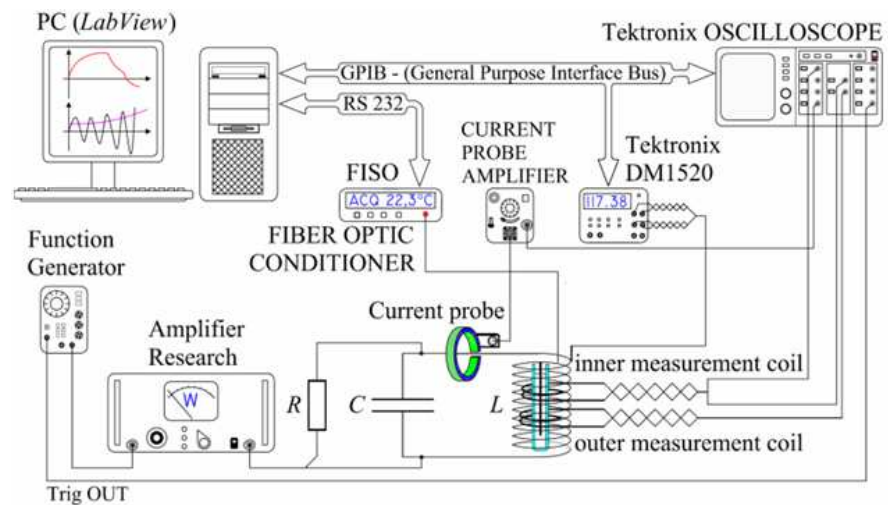

Fig. 2. Measurement scheme.

\subsubsection{Magnetic field properties}

The authors of some articles on the topic of magnetic fluid-loss characterization use those measurement systems where the measured sample is exposed to locally-different values of the magnetic fields, which means that losses are defined as the average value of a given field. In order to avoid this anomaly, the homogeneity of the field must be ensured; it can be obtained via the relatively long axial-length of the excitation coil.

This study tested different lengths or coils by increasing the number of coil turns, as show on the left side of Fig. 3. Turns of the coil were added, as seen, until the desired field deviations of less than $0.5 \%$ for amplitude $H$ had been achieved within the area housing the sample magnetic fluid. The finite-element method (FEM) software Vector Fields OPERA was used and a static FEM analysis was performed for each number of turns.
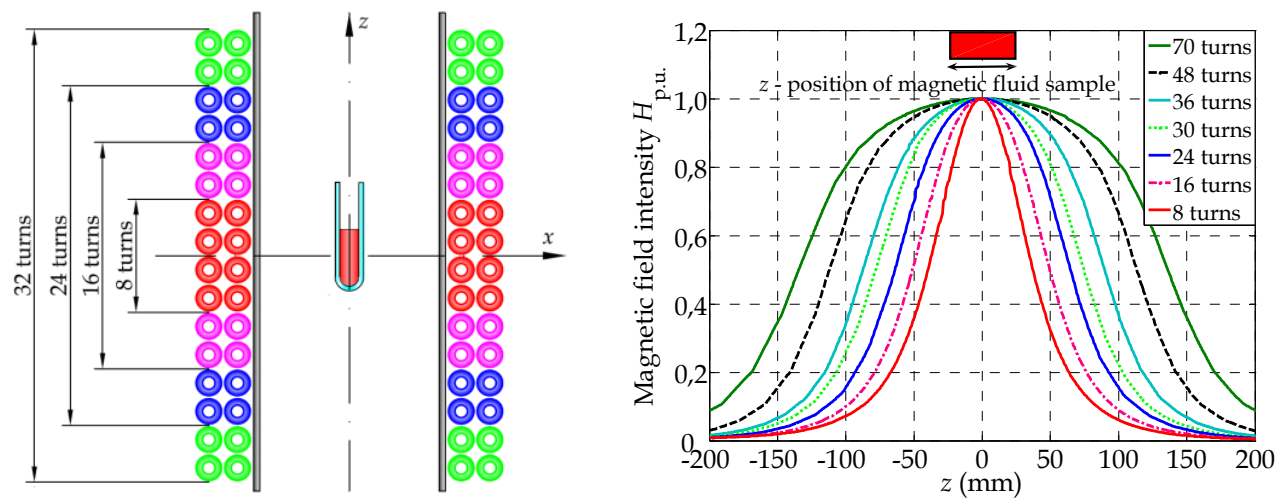

Fig. 3. Left side shows the principle of adding image excitation coil turns, right side-the calculated results of magnetic field strength along the $\mathrm{z}$-axis for different numbers of turns. 
Partial results for the FEM analyse for the seven cases of different excitation coil turns are presented at the right side of Fig. 3, showing the amplitudes of the magnetic field strengths along the $z$-axis. It can be seen that, in the case of the least coil turns, $H$ has a normal distribution. Thus it means that the sample was not exposed to the same magnetic field, both in terms of the direction and the amplitude. In this case, the deviation from the maximum value was up to $25 \%$ less, so it was necessary to add the coil-turns in order to achieve homogeneity of the magnetic field. The graph shows the result of increasing number of turns where it can be seen that adding field-coil turns reduces the deviation of the magnetic field within the position of the measured sample. A deviation of $0 \%$, would, in theory be achieved with an endless coil, therefore, a tolerance less than $0.5 \%$ in the $z$ direction was acceptable, which was achieved with 70 turns, whilst the discrepancies in the $x$ and $y$-directions were negligible.

Fig. 4 shows three examples of magnetic FEM analysis, which show the magnetic field strengths at the $z x$-plate in the centre of the measurement system. These results show that, in the case of a smaller number of turns, the magnetic field in the region of the sample is clearly non-homogeneous; in addition to archive the same value of $H$ higher values of excitation current is required and it is limited by the amplifier.

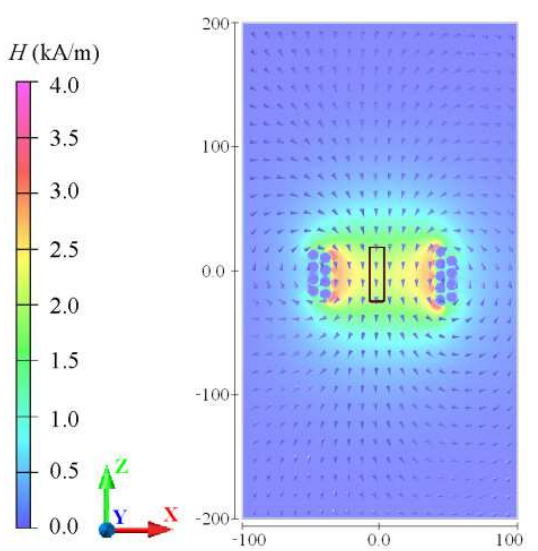

(a)

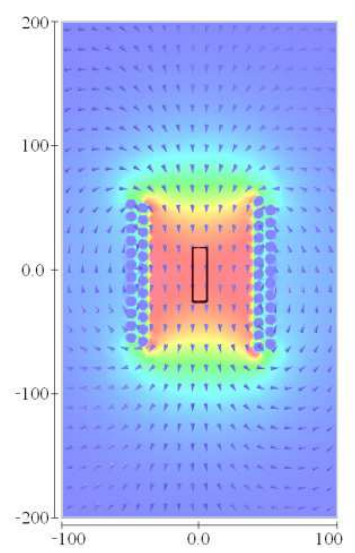

(b)

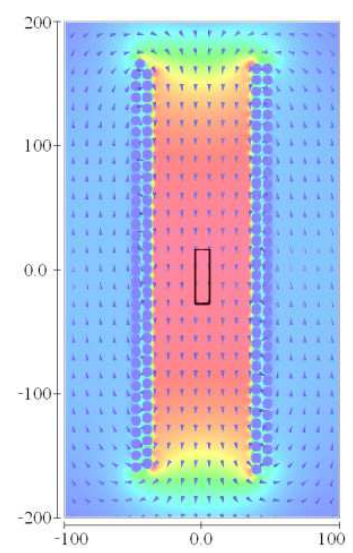

(c)

Fig. 4. Results of FEM analysis; magnetic field intensity within the measurement system for different numbers of excitation coil turns; a) 8 turns, b) 24 turns and c) 70 turns.

\subsubsection{Thermal field properties}

Besides magnetic calculation during the design process of the measurement system, a thermal analysis was preformed to ensure heat insulation within the system and the temperature stability of the sample in case of outside temperature disturbances. Several papers on magnetic fluid heating power characterization have not focused on the thermal isolation of their systems, which could result in improper temperature measurements; essential in calorimetric method. The resulting temperature increase of a sample can also be the consequence of heat transfer from the heated excitation coil. Therefore, sufficient thermal insulation must be included within the system, in such manner that any generated 
temperature increase is merely a result of the desired heating mechanism, which is the power-loss of the magnetic fluid sample.

For this part of the paper, an excitation coil with 70 turns was selected and thermal analysis for different examples performed. Fig. 5 displays part of an $x z$-cross-section of the measurement system, and the used materials. It also displays the distances or thicknesses of the materials. The first influence looked at was the impact of warming the excitation coil on the temperature of the magnetic fluid sample. The heating of the excitation coil was taken into consideration due to the Joule losses and magnetic fluid for heating caused by the aforementioned heating mechanisms. The maximum coil-current was set as given by the maximum output of power supply. By knowledge of the coil resistance, the maximum loss of excitation coils was determined together with heating power of the measuring sample. The second influence examined was the influence of the cooling water within the measuring system, since the plan was to produce a water-cooled coil. Final influence observed was the presence of a glass vacuum tube, as visible in Fig. 5 and marked within area-I and the presence of polystyrene as an insulating material on the same figure marked within area-II. Four different examples of the FEM analysis are collected on the Table. I. For this research, none of the dimensions where changed, as marked in Fig . 5 but only the presence of the materials and the water cooling of excitation coil.
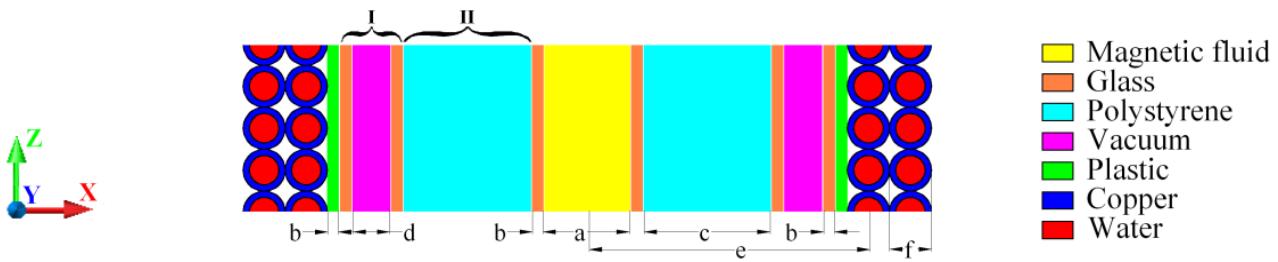

Fig. 5. The realized measurement system on the left and 3D model for thermal FEM analysis on the right.

\begin{tabular}{cccccccc}
\hline Ex. & $\begin{array}{c}\text { Heat: Mag. fluid } \\
\left(\mathrm{W} / \mathrm{mm}^{3}\right)\end{array}$ & $\begin{array}{c}\text { Heat: Excit. } \\
\text { coil } \\
\left(\mathrm{W} / \mathrm{mm}^{3}\right)\end{array}$ & $\begin{array}{c}\text { Cooling } \\
\text { Water } T \\
\left({ }^{\circ} \mathrm{C}\right)\end{array}$ & Polystyrene & $\begin{array}{c}\text { Vacuum } \\
\text { tube }\end{array}$ & $\begin{array}{c}\text { results: } \\
T_{\max }\left({ }^{\circ} \mathrm{C}\right)\end{array}$ & $\begin{array}{c}\text { results: } \\
T_{\text {avg }}\left({ }^{\circ} \mathrm{C}\right)\end{array}$ \\
\hline A & $1 \mathrm{e}^{-4}$ & $1 \mathrm{e}^{-7}$ & no & no & no & 152 & 147 \\
B & $1 \mathrm{e}^{-4}$ & $1 \mathrm{e}^{-7}$ & no & no & yes & 148 & 139 \\
$\mathrm{C}$ & $1 \mathrm{e}^{-4}$ & $1 \mathrm{e}^{-7}$ & no & yes & yes & 129 & 121 \\
$\mathrm{D}$ & $1 \mathrm{e}^{-4}$ & $1 \mathrm{e}^{-7}$ & yes & yes & yes & 110 & 108 \\
\hline
\end{tabular}

Table 1. Examples of thermal analyses.

The result for one thermal analysis for example $C$ can be seen in Fig. 6, which shows a) the temperature distribution in the measuring system on the xz-cross-section, b) the temperature derivative and c) the heat flux.

The results for all four FEM examples where the calculated temperature field distributions throughout the volume; in this case, the most interesting were displayed by the variables along the $x$-axis. The straight line was thus at the height $z=0$ and $x$ between 60 and -60 , as demonstrated in Fig. 4. 


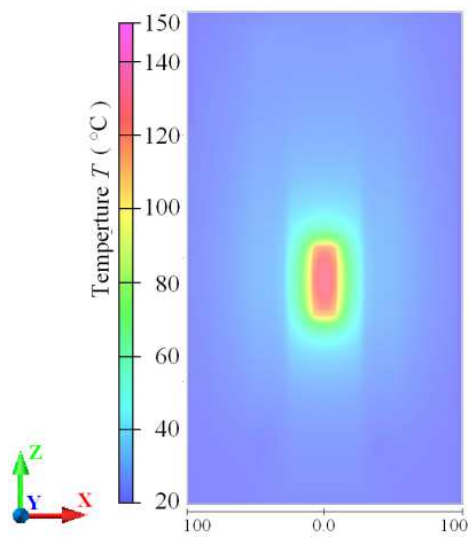

(a)

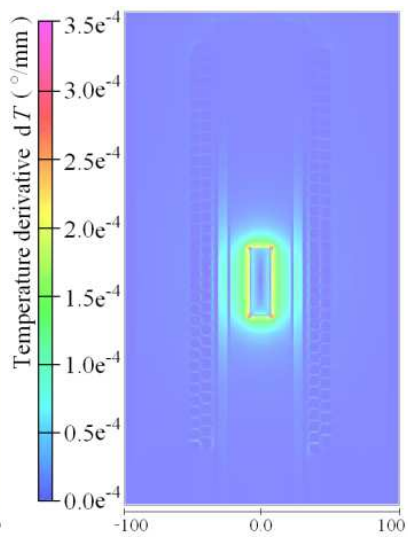

(b)

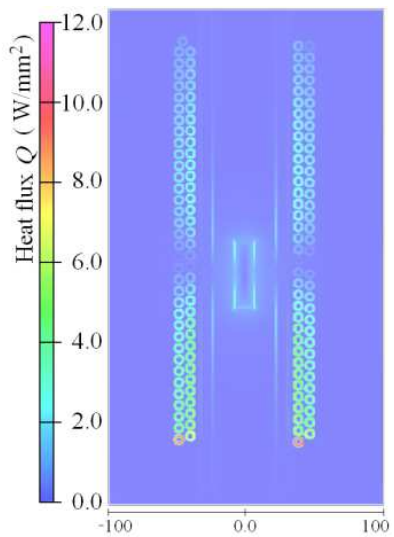

(c)

Fig. 6. Results of FEM analysis; a) the temperature distribution in the system b) the temperature derivative and c) the heat flux within the system; results obtained for ex- $\mathrm{C}$.

Figure 7 shows the results for temperature evaluated along the aforementioned line, for all four cases. It can be seen that the sample's temperature was generally higher; less of the insulation was included in the calculation. Notably in the last case, when the cooling water with the same temperature as the surroundings is included, the sample fluid is heated only because of attributed heating power, hence the lowest temperature. The impact of the ambient was minimized. When observing in the course of the heat flux (right picture) for all four cases along the same line it appeared, that in the position $40<z<50$, where the coil was located, there was a source of heat-flow directed to the outside and inside of the system, thus causing unnecessary heating of the sample. When a layer of insulation was added between the coil and the sample, heating was reduced (ex. C).
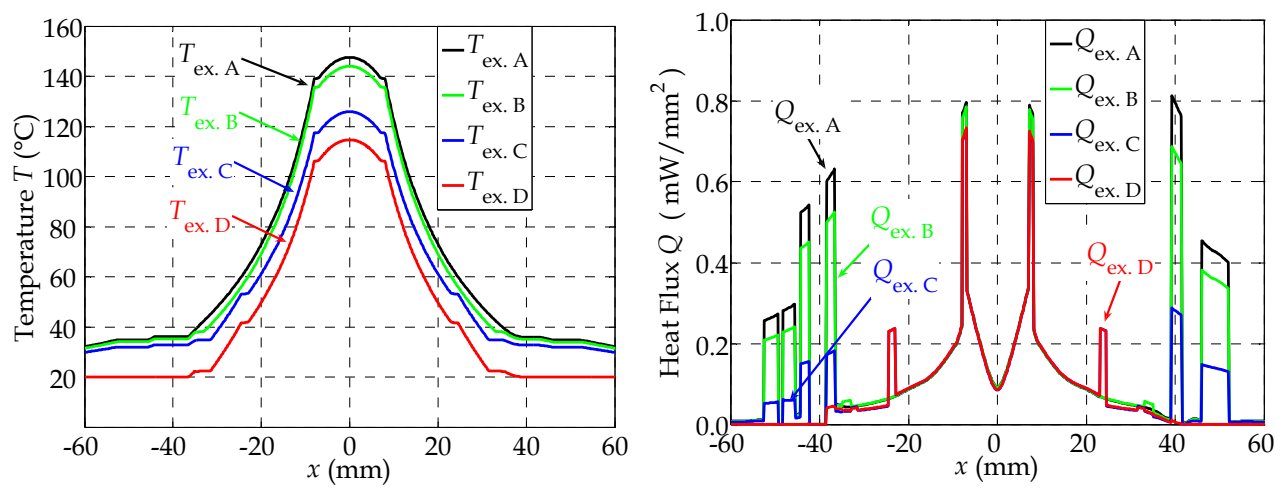

Fig. 7. Results of FEM analysis; temperature along the x-axis for examples A, B, C and D and the right picture, the heat flux along the $\mathrm{x}$-axis for the same examples. 


\subsection{The realization}

The result of this planning was a realization of the measurement system shown at the left of Fig. 8. The right picture is a $3 \mathrm{D}$ model and reveals more details, since it is partly cut out. This model was also used for all the FEM calculations presented in the previous subchapter.
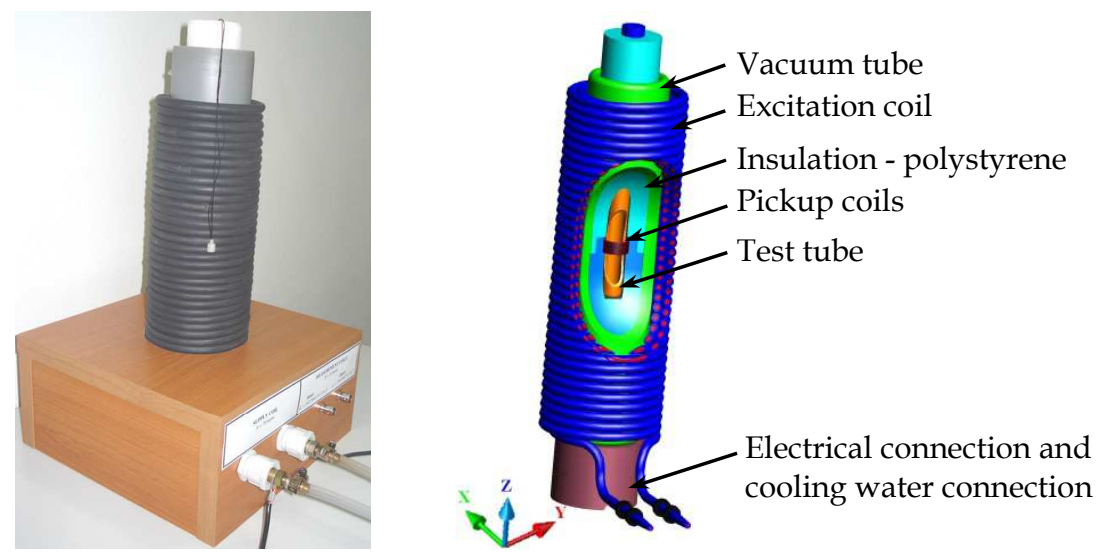

Fig. 8. The realized measurement system left side and 3D model for thermal FEM analysis right side.

\subsection{Measurements of magnetic field parameters}

So far the focus has been on the thermal insulation of the measurement system for better implementation when characterizing fluids, according to calorimetric method (3), whilst these sections have shown the principle of magnetic field variables measurement when determining the losses based on the determination of the hysteresis loop's surface (2). From the equation, it is clear that it is necessary to measure the magnetic field strength $H(t)$ and magnetic flux density $B(t)$. For this measurement a system of three measuring coils has been created, which bond to each other, as shown in Fig. 9. The top view displays the magnetic fluid sample, the middle shows around the sample containing a glass test tube for sample storage, and further outwards the two pickup coils for measuring the induced voltages $e_{1}$ and $e_{2}$. These two voltages are used in determination of magnetic field strength and magnetic flux density according to (5) and (6).

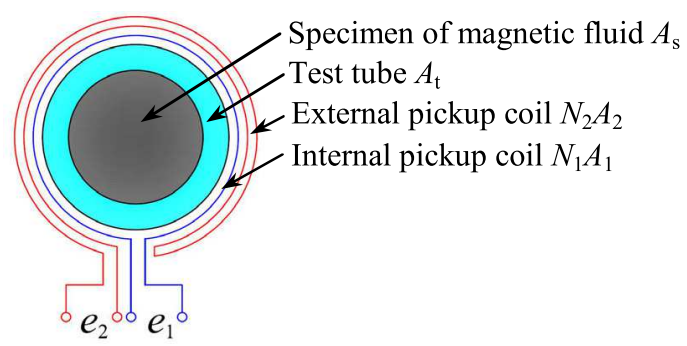

Fig. 9. Principle of measuring $B(t)$ and $H(t)$ by means of two pickup coils. 
First let's look at the magnetic flux density $B(t)$, as defined by equation (4), where $N_{1}$ is the number of inner pickup coil turns, $A_{\mathrm{s}}$ is the cross-section of the sample, and $\phi_{1}$ the magnetic flux in the sample.

$$
B(t)=\frac{\phi_{1}}{N_{1} A_{\mathrm{s}}}
$$

The inner measuring tube coil tightly embraces the vial so that the magnetic flux can be replaced by the integral of a measured induced voltage, as shown in (5).

$$
B(t)=\frac{1}{N_{1} A_{\mathrm{s}}} \int e_{1}(t) \mathrm{d} t
$$

Measurement of the magnetic field strength is a greater challenge, since the magnetic flux $\phi_{2}$ should be measured close to the sample, whilst excluding sample's magnet flux. The external pickup coil marked in Fig. 9 is used for this measurement and it is evidently wound in such a manner that embraces the sample.

$$
H(t)=\frac{1}{\mu_{0}} \frac{\phi_{2}}{N_{2} A_{2}}=\frac{1}{\mu_{0}} \frac{1}{N_{2} A_{2}} \int e_{2}(t) \mathrm{d} t
$$

Equation (6) contains $\mathrm{N}_{2}$ and $A_{2}$ which are constants and represent the number of coil turns and its cross-section. It was determined experimentally in such a manner, that the magnetic flux linkages where measured whilst exposing the coil to the known density of the magnetic field.

\section{Testing}

This section verifies the operation of the measuring system on a sample of magnetic fluid. Firstly, the calorimetric method is described and then those methods based on the magnetic field quantities measurements.

The magnetic fluid sample used in this work is commercially available; therefore some tests were performed to determine the basic structural properties of the fluid. Chemical analysis revealed particles of maghemite $\gamma-\mathrm{Fe}_{2} \mathrm{O}_{3}$ dispersed in mineral oil. Transmission electron microscopy revealed the mean diameter of particles to be $10.9 \mathrm{~nm}$. Magnetic measurement of its magnetization curve was performed and modified according to the Langevine theory of paramagnetism (Pshenichnikov) the magnetization of particles, close to saturation, is described by

$$
M=M_{\mathrm{s}}\left(1-\frac{k_{\mathrm{B}} T}{\mu_{0} M_{\text {s bulk }} \pi / 6 d_{\text {core }}^{3}} \frac{1}{H}\right),
$$

The volume fraction of the magnetic particles in the used sample of magnetic fluid was determined from a ration of $M_{\mathrm{s}} / M_{\mathrm{s}}$ bulk, where $M_{\mathrm{s}}$ bulk was $400 \mathrm{kA} / \mathrm{m}$ and it is $10.57 \%$.

\subsection{Magnetic fluid characterization SAR}

Calorimetric measurements were carried out using a calorimeter, which is an instrument that allows the heat-effects within it to be determined by direct measurement of 
temperature, as proposed in (1). The sample of magnetic fluid was exposed to an ac magnetic field. The measurement principle for the calorimetric measurements required a stable temperature of the sample, before the magnetic field could be switched on. For this reason, the measurement could only begin when the temperature of the sample was constant, usually at the value of cooling water. When this condition had been fulfilled, the excitation coil was turned on and constant frequency and regulated current set up the magnetic field at the desirable value. The measurement lasted until the temperature steady state had been reached or if the other heat production equalled the heat dissipation.

Several tests, for an $8 \mathrm{ml}$ sample of magnetic fluid, of different magnetic field strengths were performed and the results for temperature rise are presented in Fig. 10. The measurements were performed at a frequency of $400 \mathrm{kHz}$ and constant field amplitudes, that were regulated to achieve a discrepancy of $\pm 10 \mathrm{~A} / \mathrm{m}$ around the selected values.
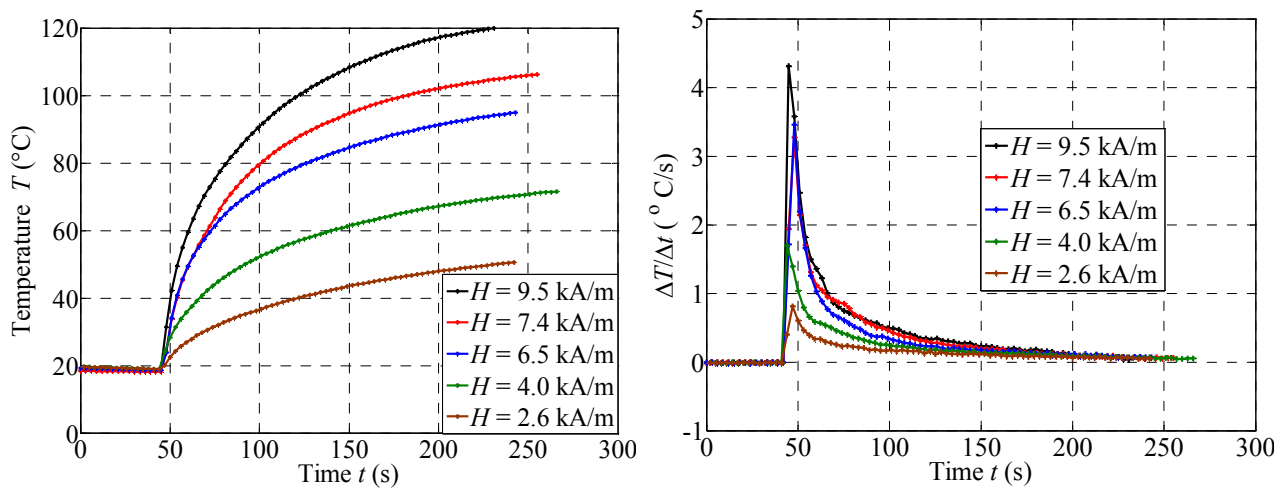

Fig. 10. Calorimetric measurements of temperature rise for different values of magnetic field strengths at frequency of the supply field of $400 \mathrm{kHz}$, and the temperature derivatives.

This approach is not new regarding magnetic fluid determination, but it serves as a verification method for determining losses through hysteresis loops or through complex susceptibility, as presented below.

\subsection{Magnetic fluid characterization SPL}

This section explores the methods for determining the losses of magnetic fluids at constant temperature. For this, equation (3) was used to calculate the losses from the area of the hysteresis loop and, in the second case, out of a complex susceptibility, as explained later, both based on the measurement of time courses $H(t)$ from equation (6) and $B(t)$ from (5). In the analysis, magnetic polarization was used instead of magnetic flux density, where

$$
B=J+\mu_{0} H
$$

Another approach for determining losses is based on (9) according to Carrey, where the parameter of the complex susceptibility $\chi^{\prime \prime}$ represents the essential component

$$
P_{\chi}=\frac{1}{\rho} \pi \mu_{0} H^{2} \chi^{\prime \prime} f
$$


This section shows the equivalences of these methods for the sampling the magnetic fluid and point out the advantages of these methods against the calorimetric method. In this case, the characterization losses are executed under such conditions as would be applied during actual treatment with hyperthermia.

The relationship between relative permeability and susceptibility was determined using this expression

$$
\mu_{\mathrm{r}}=\frac{B}{\mu_{0} H}=\frac{J+\mu_{0} H}{\mu_{0} H}=\frac{J}{\mu_{0} H}+1=\chi+1
$$

The responses of the material to the applied magnetic field were studied using the complex susceptibility approach. In this case the susceptibility was treated as a real $\chi^{\prime}$ component, which is within the phase with the magnetic field and imaginary component $\chi^{\prime \prime}$ that lags behind the $90^{\circ}$, the latter is also related to the energy losses or absorbed energy by the sample from the ac field respectively. The terms for both were written (11), where $\delta$ was the angle between the measured signals $H(t)$ and $J(t)$ or $B(t)$

$$
\chi=\frac{J}{\mu_{0} H} \Rightarrow \chi^{\prime}=\frac{\hat{J}}{\mu_{0} \hat{H}} \cos \delta, \chi^{\prime \prime}=\frac{\hat{J}}{\mu_{0} \hat{H}} \sin \delta,
$$

The characterization was carried out in such a manner that different values for power amplifier currents were set, and thus the magnetic field strength whilst measuring the voltages of the two pickup coils. Such an example occurs in the lower two graphs showing the courses of the induced voltages $e_{1}$ and $e_{2}$ for the change of gain over seventeen steps.
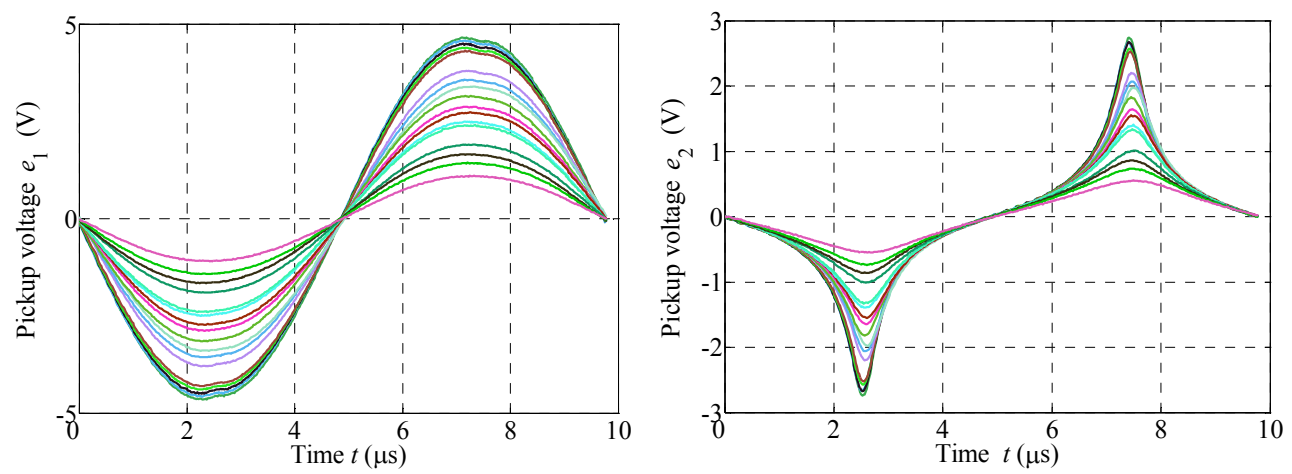

Fig. 11. Measured induced voltages $e_{1}$ and $e_{2}$ for various examples of magnetic fields between 5 and $15 \mathrm{kA} / \mathrm{m}$.

Here it should be noted that the measurements must be carried out within a very short period of time, since a long exposure of the sample to the high value of the field quickly rises the temperature, as is clear from the heat curves in Fig. 10. Magnetic fluid at higher temperatures behaves differently, since it's the closer to Curie's temperature which also decreases its heating power because of the narrower hysteresis loop. From the measured induced voltage the desired variables $H(t)$ and $B(t)$ can be calculated, which are plotted in Fig 12 for the same example. 

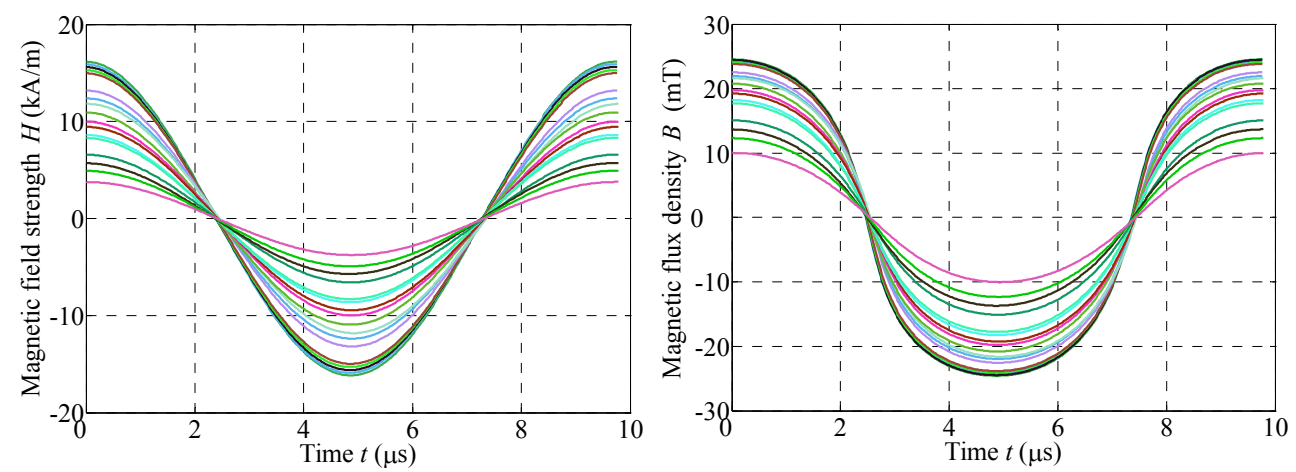

Fig. 12. The time courses of the calculated values $H(t)$ and $B(t)$ for the same example.

The measured signals provide the determination of losses using both methods, namely by determining the hysteresis loop area using equation (2) or through the determination of the complex susceptibility and losses calculated by equation (9). Fig. 13 below shows the two approaches, the left images are the outlined $J H$ hysteresis loops for determining the losses by the first method. Determination of the surface hysteresis is no novelty, but an interesting approach for the evaluation of the dynamic hysteresis loop areas of super paramagnetic materials, and the determination of loss by this method.

The right side figure shows the magnetic filed $\mu_{0} H$ box and the response of materials to the field of magnetic polarization $J(t)$ (you could also use $B(t)$ ). The goal was to determine the phase shifts of signals $\delta$ to be able to determine the $\chi^{\prime \prime}$ according to (11).
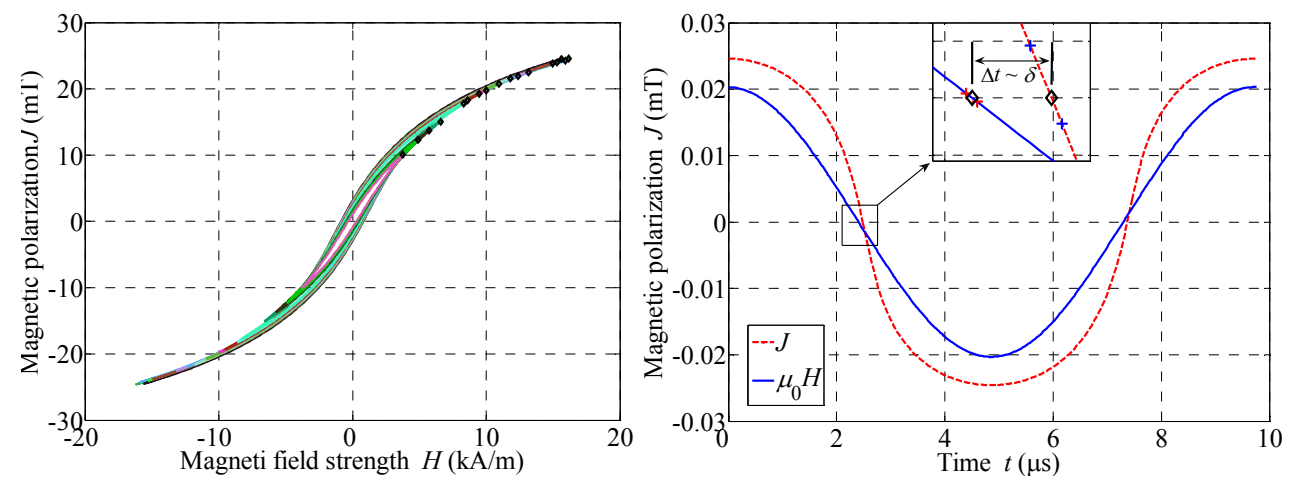

Fig. 13. Hysteresis loop and the time course of signals for determining magnetic losses.

If the plot of the phase shifts in delta is now done for all seventeen cases, it is found that only the latter is not constant but decreases linearly, as shown in Fig. 14. According to the formula for the losses determined from a complex susceptibility $P_{X}$ (9) the desired characteristic $P=f(H)$ can be calculated, which is for example of the $100 \mathrm{kHz}$ frequency given in the same graph. 


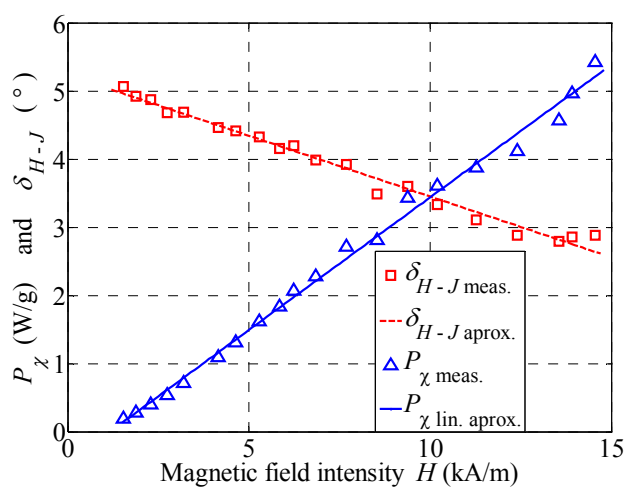

Fig. 14. The result of the phase shift $\delta$ measurements for different magnetic field strengths and associated loss of magnetic fluid sample at $100 \mathrm{kHz}$.

When the losses of $P_{\mathrm{h}}$ were plotted in the same graph as the loss of $P_{\mathrm{X}}$ it was evident, that both analyses of the measured signals offered the same results. The results are summarized in Fig. 15 for four different frequencies of the applied field and it was discovered that the losses expectedly increased when increasing the frequencies.

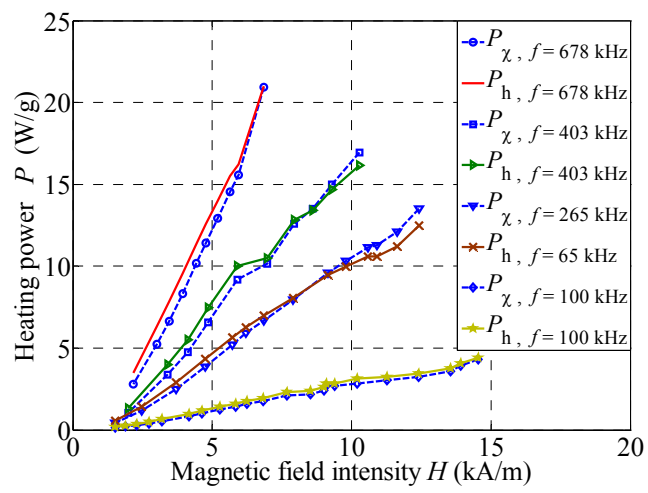

Fig. 15. A comparison of heating powers $P_{\chi}$ and $P_{\mathrm{h}}$ for four different frequencies, and the amplitudes of magnetic field intensity.

\section{Conclusion}

In conclusion we can point out specific guidelines that apply to measuring systems for determining the losses of magnetic fluids. In this context, the losses are a desired property, because it means greater heat output and thus higher quality material for usage in medical hyperthermia.

In order to build such a measurement system it is necessary firstly to provide a source magnetic field that must be able to change both the amplitude and frequency of the magnetic field. In achieving this objective, we have tried to approximate as closely as possible those fields parameters that would actually be best suited for implementing this treatment. 
Secondly, we need a measurement system which is sufficiently precise and will allow for a clear characterization of magnetic fluid losses. This article presents three methods, where each of them exposed their weakness, and the possibility of erroneous measurements.

In the calorimetric method, the emphasis is on adiabatic changes in temperature, at least at the start of the measurement, which determines the maximum slope of warming. For FEM analysis, we have demonstrated the importance of certain measures for improving the methodology.

The method of magnetic measurement emphasizes the accuracies of the measuring coil parameters, since only with precisely defined parameters can accurate measurements be performed. We here shown the equivalence of both methods, where we see that the determination of the hysteresis loop area and determination of the angle between the polarization and the magnetic field brings almost the same result for determining the losses, depending on the accuracies of certain constants.

\section{References}

Barba, P., Dughiero, F., \& Seini, E. (2010). Magnetic field synthesis in the design of inductors for magnetic fluid hyperthermia. IEEE Transaction on Magnetics, Vol. 46, No. 8, pp. 2931-2943, 0018-9464.

Beković, M., \& Hamler, A. (2010). Determination of the heating effect of magnetic fluid in alternating magnetic field. IEEE Transaction on Magnetics, Vol. 46, No.2, pp. 552-555, 0018-9464.

Carrey, J., Mehdaoui, B., \& Respaud, M. (2011). Simple models for dynamic hysteresis loop calculations of magnetic single-domain nanoparticles: Application to magnetic hyperthermia optimization. Journal of Applied Physics 109, 083921-1-17, 0021-8979.

Jeong, J. R., Lee, S. J., Kim, J. D., \& Shin, S. C. (2004), Magnetic properties of $\mathrm{Fe}_{3} \mathrm{O}_{4}$ nanoparticles encapsulated with poly (D,L Lactide-Co-Glycolide). IEEE Transaction on Magnetics, Vol. 40, No.4, pp. 3015-3017, 0018-9464.

Jibin, Z., \& Yongping L., (1992). Numerical calculations for ferrofluid seals. IEEE Transaction on Magnetics vol. 28, No.6, pp. 3367 - 3371, 00189464.

Kim, D. H., Thai, Y. T., Nikels, D. E., \& Brazel, C. S., (2009). Heating of aqueous dispersions containing $\mathrm{MnFe} 2 \mathrm{O} 4$ nanoparticles by radio-frequency magnetic field induction, IEEE Transaction on Magnetics, Vol. 45, No.1, pp. 64-70, 0018-9464.

Kumar, C (Ed(s)). (2009). Magnetic Nanoparticles, nanomaterials for life science Volume 4, Wiley-vch Verlag GmbH\&Co.

Pavel, M., \& Stancu, A. (2009). Ferromagnetic nanoparticles dose based on tumor size in magnetic fluid hyperthermia cancer therapy. IEEE Transaction on Magnetics, Vol. 45, No.11, pp. 5251-5254, 0018-9464.

Pshenichnikov A.F., Mekhonoshin V.V., \& Lebedev A.V., “Magneto-granulometric analysis of concentrated ferrocolloids", J. Magn. Magn. Mater. 161, pp. 94-102, 1996.

Pollert, E., Knižek, K., Maryško, M., Kašpar, P., Vasseur, S., \& Duguet, E., (2007), New T ${ }^{-}$ tuned magnetic nanoparticles for self-controlled hyperthermia. Journal of Magnetism and Magnetic Materials, 316, pp. 122-125, 0304-8853.

Rosensweig, R.E., "Ferrohydrodynamics”, Dover publications, inc, Mineola New York 1997. 


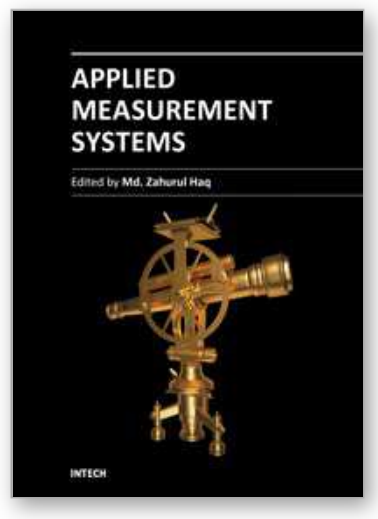

\author{
Applied Measurement Systems \\ Edited by Prof. Zahurul Haq
}

ISBN 978-953-51-0103-1

Hard cover, 390 pages

Publisher InTech

Published online 24, February, 2012

Published in print edition February, 2012

Measurement is a multidisciplinary experimental science. Measurement systems synergistically blend science, engineering and statistical methods to provide fundamental data for research, design and development, control of processes and operations, and facilitate safe and economic performance of systems. In recent years, measuring techniques have expanded rapidly and gained maturity, through extensive research activities and hardware advancements. With individual chapters authored by eminent professionals in their respective topics, Applied Measurement Systems attempts to provide a comprehensive presentation and in-depth guidance on some of the key applied and advanced topics in measurements for scientists, engineers and educators.

\title{
How to reference
}

In order to correctly reference this scholarly work, feel free to copy and paste the following:

Miloš Beković and Anton Hamler (2012). Experimental System for Determining the Magnetic Losses of Super Paramagnetic Materials; Planning, Realization and Testing, Applied Measurement Systems, Prof. Zahurul Haq (Ed.), ISBN: 978-953-51-0103-1, InTech, Available from: http://www.intechopen.com/books/appliedmeasurement-systems/experimental-system-for-determining-the-magnetic-losses-of-superparamagneticmaterials-planning-real

\section{INTECH}

open science | open minds

\author{
InTech Europe \\ University Campus STeP Ri \\ Slavka Krautzeka 83/A \\ 51000 Rijeka, Croatia \\ Phone: +385 (51) 770447 \\ Fax: +385 (51) 686166 \\ www.intechopen.com
}

\author{
InTech China \\ Unit 405, Office Block, Hotel Equatorial Shanghai \\ No.65, Yan An Road (West), Shanghai, 200040, China \\ 中国上海市延安西路65号上海国际贵都大饭店办公楼 405 单元 \\ Phone: +86-21-62489820 \\ Fax: +86-21-62489821
}


(C) 2012 The Author(s). Licensee IntechOpen. This is an open access article distributed under the terms of the Creative Commons Attribution 3.0 License, which permits unrestricted use, distribution, and reproduction in any medium, provided the original work is properly cited. 